\title{
Convergence of the hyperspherical-harmonics expansion with increasing number of particles for bosonic systems. II. Inclusion of the three-body force
}

\author{
N. K. Timofeyuk \\ Faculty of Electronics and Physical Sciences, Physics Department, University of Surrey, Guildford, \\ Surrey GU2 7XH, United Kingdom \\ (Received 16 March 2015; published 30 April 2015)
}

\begin{abstract}
This paper presents a numerical convergence study of a hyperspherical-harmonics expansion for binding energies of a system of $4 \leqslant N \leqslant 728$ helium atoms using a phenomenological soft attractive two-body He-He potential and a repulsive three-body force aimed at compensating for the absence of the two-body repulsive core. Earlier calculations with such a potential have shown an improved convergence when $N$ increases from four to six. The present study reveals that the improved convergence occurs only for a limited range of $N$ determined by the range of the three-body repulsion. For a soft repulsive three-body force, the convergence is fast for $N \leqslant 20$, while for a short-range three-body repulsion it deteriorates at $N \geqslant 10$. The reasons for this deterioration are discussed. The range of the three-body force also determines the binding energy behavior with $N$, and it is also responsible for binding the excited states. The long-range force binds all first excited $0^{+}$states but strongly underbinds the systems of $N$ helium atoms at large $N$. The short-range force does not bind the first $0^{+}$states for $A \leqslant 7$ but gives better predictions of binding energies as compared to the calculations of other authors though overestimating them. Some options to improve both the description of the binding energies and the convergence of the hyperspherical-harmonics expansion using phenomenological forces are discussed. It is pointed out that a fast convergence is very much needed for the reliable predictions of states with nonzero angular momentum, examples of which are also given.
\end{abstract}

DOI: 10.1103/PhysRevA.91.042513

PACS number(s): 31.15.xj, 31.15.xt

\section{INTRODUCTION}

In a previous paper [1] I was trying to understand the reason for improved convergence of hyperspherical-harmonics expansion with increasing number of bosons observed in calculations of binding energies for four, five, and six helium atoms in Ref. [2]. These calculations used a simple twobody interaction and predict the correct value for the $\mathrm{He}-\mathrm{He}$ scattering length and is represented by only one Gaussian, and a three-body He-He-He potential of the hypercentral form with the radius and the depth fitted to reproduce the realistic calculations of binding energies for four, five, and six helium atoms from other many-body methods. The convergence of the hyperspherical expansion for six atoms in Ref. [2] was better than that for four atoms both when only the two-body force was used and when the three-body force was added. This observation is important since it suggests that in the limit of a large scattering length a fast solution of the many-body problem could be achieved by using a simple phenomenological soft two-body potential plus a repulsive three-body force accounting for missing correlations between the constituents of the many-body system.

In Ref. [1], it has been shown that the reason for the improved convergence with increasing boson number, observed in Ref. [2], originates from the decrease of the ratio of nondiagonal coupling potential to the lowest-order diagonal potential, associated with a single Gaussian potential, as $N^{1 / 2}$ or faster at $N \rightarrow \infty$. The improved convergence has been further confirmed by numerical calculations, performed with the two-body potential from Ref. [2] presented by a single Gaussian. However, this convergence could be affected in the presence of the repulsive core, presented by a second Gaussian, when nondiagonal couplings to higher order hyperradial potentials are strong near the node of the lowest order hyperradial potential. Several examples of a slower convergence due to a second Gaussian in the two-body potential have been presented in Ref. [1]. The same picture holds when a three-body hypercentral potential of a simple Gaussian form is added to the scheme. The ratio of nondiagonal to the lowest diagonal hyperradial potential, associated with this term only, also decreases with $N$ as $N^{-1 / 2}$ or faster [1]. However, as the strength of the contribution from three-body repulsion increases faster with $N$ than that of the two-body attraction, starting from some $N$ the total diagonal hyperradial potential develops a strong repulsive core pushing the attractive potential well into large hyperradii. The magnitude of this core is much larger than the depth of the attractive potential well. The strong core also develops in (much smaller) nondiagonal coupling hyperradial potentials, and the tail from these nondiagonal couplings can be nonnegligeable near the node of the lowest order hyperradial potential, which can affect the convergence.

When the work in Ref. [1] was carried out, the hyperspherical-harmonics expansion code calculated the contribution from the three-body force only in the lowest order approximation, and, therefore, the numerical investigation of the convergence of hyperspherical expansion with $N$ could not be carried out. Nevertheless, it was suggested there that the hyperspherical-harmonics expansion convergence should improve with $N$, following the trend observed in Ref. [2]. Recently the three-body force has been included into this code for any hypermomenta, and the code has been validated against the energies of four-, five-, and six-body systems published in Ref. [2], providing an agreement with all five digits published there. The present paper reports the results of the convergence study of the hyperspherical expansion with increasing $N$, performed using the new code, for two sets of two- and three-body potentials which predict the same binding energies of the two- and three-body systems but 
have a different range of the three-body repulsion. In Sec. II the hypersperical formalism is outlined. Section III explains the choice of the interaction potentials and methods used to solve the hyperradial equations. The binding energies for $0^{+}$states of several systems helium clusters are presented in Sec. IV as a function of the model space, while the binding energies of the states with nonzero angular momentum are studied in Sec. IV. Section V summarizes and discusses the results obtained.

\section{HYPERSPHERICAL FORMALISM}

We start with the Hamiltonian

$$
H=\hat{T}+\sum_{1 \leqslant i<j \leqslant N} V_{i j}+\sum_{1 \leqslant i<j<k \leqslant N} W_{i j k}
$$

and will solve the eigenvalue problem $H \Psi=E \Psi$ in hyperspherical coordinates $\{\rho, \hat{\rho}\}$, where $\rho$ is the hyperradius and $\hat{\rho}$ are hyperangles [3]. In the hyperspherical-harmonics method, the $N$-body wave function $\Psi(\rho, \hat{\rho})$ is presented as an expansion

$$
\Psi=\rho^{-(3 N-4) / 2} \sum_{K \gamma}^{K_{\max }} \chi_{K \gamma}(\rho) Y_{K \gamma}(\hat{\rho})
$$

onto a complete set of eigenfunctions $Y_{K \gamma}(\hat{\rho})$ of the angular part of the $3 N-4$-dimensional Laplacian $\Delta_{\hat{\rho}}$ [3], where the quantum number $K$ is a hyperangular momentum and the index $\gamma$ denotes the set of all other possible quantum numbers. The hyperradial functions $\chi_{K \gamma}(\rho)$ are found from the solution of the coupled set of differential equations

$$
\begin{aligned}
& {\left[\frac{d^{2}}{d \rho^{2}}-\frac{\mathcal{L}_{K}\left(\mathcal{L}_{K}+1\right)}{\rho^{2}}-\frac{2 m}{\hbar^{2}}\left[E+U_{K \gamma, K \gamma}(\rho)\right]\right] \chi_{K \gamma}(\rho)} \\
& \quad=\frac{2 m}{\hbar^{2}} \sum_{K^{\prime} \gamma^{\prime} \neq K \gamma} U_{K \gamma, K^{\prime} \gamma^{\prime}}(\rho) \chi_{K^{\prime} \gamma^{\prime}}(\rho),
\end{aligned}
$$

where $\mathcal{L}_{K}=K+(3 N-6) / 2, m$ is the boson mass, and the hyperradial potentials $U_{K \gamma, K^{\prime} \gamma^{\prime}}(\rho)$,

$$
U_{K \gamma, K^{\prime} \gamma^{\prime}}(\rho)=V_{K \gamma, K^{\prime} \gamma^{\prime}}(\rho)+W_{K \gamma, K^{\prime} \gamma^{\prime}}(\rho),
$$

are the matrix elements of the two- and three-body interactions:

$$
\begin{aligned}
& V_{K \gamma, K^{\prime} \gamma^{\prime}}(\rho)=\left\langle Y_{K \gamma}(\hat{\rho})\left|\sum_{i<j} V_{i j}\right| Y_{K^{\prime} \gamma^{\prime}}(\hat{\rho})\right\rangle, \\
& W_{K \gamma, K^{\prime} \gamma^{\prime}}(\rho)=\left\langle Y_{K \gamma}(\hat{\rho})\left|\sum_{i<j<k} W_{i j k}\right| Y_{K^{\prime} \gamma^{\prime}}(\hat{\rho})\right\rangle .
\end{aligned}
$$

The hyperspherical harmonics $Y_{K \gamma}(\hat{\rho})$ are constructed here from symmetrized products of the single-particle oscillator wave functions using the method developed in Refs. [4,5]. In this method the calculation of matrix elements is reduced to evaluation of the Laplace transforms of the standard two- and three-body matrix elements arising in the harmonic-oscillatorbased configuration interaction method.

\section{POTENTIAL CHOICE AND SOLUTION OF HYPERRADIAL EQUATIONS}

The convergence of the binding energies has been investigated for many-body systems made of helium atoms assuming a simple Gaussian form for the two- and the three-body interactions:

$$
V\left(r_{i j}\right)=V_{0} e^{-r_{i j}^{2} / r_{0}^{2}}, \quad W\left(\rho_{i j k}\right)=W_{0} e^{-2 \rho_{i j k}^{2} / \rho_{0}^{2}},
$$

where $r_{i j}$ is the distance between particles $i$ and $j$ and $\rho_{i j k}^{2}=$ $\frac{2}{3}\left(r_{i j}^{2}+r_{j k}^{2}+r_{k i}^{2}\right)$ is the three-body hyperradius. Two sets of potentials were used:

(A) $V_{0}=-1.227 \mathrm{~K}, r_{0}=10.03$ a.u., $W_{0}=0.442 \mathrm{~K}$, and $\rho_{0}=14$ a.u. This potential has been fitted in Ref. [2] to reproduce the binding energies of four, five, and six helium atoms. It has a large range for the three-body repulsive force chosen on the assumption that it is related to the range of the two-body potential. At large scattering lengths no information is available about the two-body potential at the interatom distances smaller than the range of their interaction $R$. Therefore, one could expect that no information about three-body force should also be given for three-body distances, presented by a hyperadius, smaller than $\sqrt{2} R$, which determines the radius $\rho_{0}$ [2]. Although set A gives a reasonable description for $N \leqslant 6$, it severely underbinds the helium systems for larger $N$ in the lowest order hyperspherical-harmonics expansion [1], thus suggesting that the full converged energies, calculated with this potential, would also be too small.

(B) $V_{0}=-1.2343566 \mathrm{~K}, r_{0}=10$ a.u., $W_{0}=18 \mathrm{~K}$, and $\rho_{0}=5.8$ a.u. This potential, used in Ref. [1], has a significantly smaller range, which leads to larger binding energies for $N>6$. In the $K_{\max }=0$ approximation, it gives for $N \leqslant 112$ the binding energies similar to ab initio results obtained by several methods [7-9]. Interestingly, this range is very close to the range of the "universal" repulsive three-body force given by $2 R_{\mathrm{vdW}}$ [6], where $R_{\mathrm{vdW}}$ is the van der Waals length that for helium multimers equals $1.4 \AA$. It was hoped in Ref. [1] that the binding energies would not change much if $K_{\max }$ increases, which is a subject to verification in the present paper.

With the chosen interaction and with $\hbar^{2} / m=43.281307$ (a.u. $)^{2} \mathrm{~K}$ the coupled system of hyperradial equation (3) has been solved using two methods, (i) a finite difference scheme and (ii) expansion of $\rho^{-\frac{3 N-4}{2}} \chi_{K \gamma}$ into complete sets of $3 N-4$ dimensional harmonic oscillator wave functions, the explicit form of which is given in Refs. [4,5]. The integration step and the range of $\rho$ in the first method have been chosen to provide stable solutions that agree with those obtained by the harmonic oscillator expansion method. The latter uses 12 to 20 basis states to provide independence of the energies to the choice of the oscillator radius. For most cases, the agreement within five digits between solutions obtained by the two methods has been achieved. It was found that with increasing $N$ the accuracy of the harmonic oscillator expansions deteriorates because of the loss of precision in calculating the Laguerre polynomials; therefore, only results obtained within the finite difference method are presented below for such cases.

\section{CONVERGENCE FOR $0^{+}$STATES}

The binding energies $E_{0}$ and $E_{1}$ of the first two $0^{+}$ states for $N=4,10,20,112,240$, and 728 , calculated for $K_{\text {max }} \leqslant 14$, are shown in Table I. They have been chosen because of the availability of several $a b$ initio calculations, such as diffusion Monte Carlo [7], Monte Carlo hyperspherical calculations [8], variational Monte Carlo, and Green's 
TABLE I. Absolute values of binding energies (in K) for a system of $N$ helium atoms obtained with the potential sets A and B (see Sec. III) for several values of $K_{\max }$. The energies for $N=4$ are the same as in Ref. [2].

\begin{tabular}{|c|c|c|c|c|c|c|c|c|c|c|c|c|}
\hline \multirow[b]{2}{*}{$K_{\max }$} & \multicolumn{2}{|c|}{$N=4$} & \multicolumn{2}{|c|}{$N=10$} & \multicolumn{2}{|c|}{$N=20$} & \multicolumn{2}{|c|}{$N=112$} & \multicolumn{2}{|c|}{$N=240$} & \multicolumn{2}{|c|}{$N=728$} \\
\hline & $E_{0}$ & $E_{1}$ & $E_{0}$ & $E_{1}$ & $E_{0}$ & $E_{1}$ & $E_{0}$ & $E_{1}$ & $E_{0}$ & $E_{1}$ & $E_{0}$ & $E_{1}$ \\
\hline & & & & & Set A & & & & & & & \\
\hline 0 & 0.53893 & 0.00456 & 7.6393 & 6.0258 & 22.286 & 20.551 & 153.29 & 152.05 & 336.13 & 335.14 & 1037.0 & 1036.3 \\
\hline 4 & 0.56169 & 0.04029 & 7.6865 & 6.2053 & 22.544 & 20.981 & 157.89 & 156.60 & 344.25 & 343.22 & 1053.0 & 1052.3 \\
\hline 6 & 0.56678 & 0.06747 & 7.6928 & 6.2383 & 22.657 & 21.115 & 160.45 & 159.15 & 349.91 & 347.88 & 1062.5 & 1061.8 \\
\hline 8 & 0.56821 & 0.08422 & 7.6953 & 6.2806 & 22.708 & 21.224 & 162.88 & 161.57 & 353.31 & 352.27 & 1071.4 & 1070.6 \\
\hline 10 & 0.56858 & 0.09604 & 7.6958 & 6.2953 & 22.727 & 21.275 & 165.10 & 163.76 & 357.47 & 356.41 & 1079.9 & 1079.2 \\
\hline 12 & 0.56873 & 0.10530 & 7.6959 & 6.3061 & 22.735 & 21.310 & 167.07 & 165.72 & 361.23 & 360.16 & 1087.8 & 1087.0 \\
\hline 14 & 0.56877 & 0.11117 & 7.6959 & 6.3115 & $\begin{array}{r}22.738 \\
\text { Set B }\end{array}$ & 21.324 & 168.86 & 167.48 & 364.71 & 363.62 & 1095.1 & 1094.4 \\
\hline 0 & 0.55318 & 0.00212 & 8.3501 & 6.3206 & 31.316 & 28.697 & 402.28 & 399.42 & 1059.9 & 1057.3 & 3903.0 & 3900.9 \\
\hline 4 & 0.57656 & 0.0385 & 8.6612 & 6.8006 & 32.940 & 30.212 & 417.93 & 414.93 & 1090.1 & 1087.4 & 3969.2 & 3867.1 \\
\hline 6 & 0.58293 & 0.0672 & 8.8642 & 6.9783 & 34.038 & 31.208 & 426.42 & 423.38 & 1106.8 & 1104.1 & 4007.3 & 4005.1 \\
\hline 8 & 0.58527 & 0.0848 & 9.0406 & 7.1331 & 34.976 & 32.156 & 434.56 & 431.46 & 1122.7 & 1120.0 & 4043.2 & 4041.0 \\
\hline 10 & 0.58626 & 0.09527 & 9.1677 & 7.2186 & 35.791 & 32.956 & 441.81 & 438.65 & 1137.4 & 1134.6 & 4077.4 & 4075.2 \\
\hline 12 & 0.58670 & 0.10446 & 9.2437 & 7.3043 & 36.518 & 33.677 & 448.26 & 445.05 & 1150.5 & 1147.7 & 4108.5 & 4106.3 \\
\hline 14 & 0.58692 & 0.11274 & 9.2851 & 7.3530 & 37.161 & 34.298 & 454.07 & 450.81 & 1162.5 & 1159.7 & 4137.5 & 4135.3 \\
\hline
\end{tabular}

functions Monte Carlo [9], for these systems. One can see that for $N=10$ the convergence of $E_{0}$ and $E_{1}$, calculated with potential A, is much improved as compared to the $N=4$ case, in agreement with expectations in Refs. [1,2]. A similar improvement is observed for $N=7,8$, and 9 (not shown here). Both the binding energies $E_{0}$ and $E_{1}$ in this region are practically converged by $K_{\max }=14$. However, at $N=20$ the convergence of $E_{0}$ has slightly slowed while for $E_{1}$ still remaining better than for the $N=4$ case. At $N=112$ the convergence deteriorates for both the ground and excited states and $K_{\max }=14$ is not sufficient anymore. Exponential extrapolation suggests that $E_{0} \sim-186 \mathrm{~K}$ and $E_{1} \sim-179 \mathrm{~K}$, which is about $20 \%$ away from the $K_{\max }=0$ value, further than was expected in Ref. [1]. A similar situation occurs with $N=240$ and $N=728$ where the extrapolated values are about $20 \%$ and $15 \%$ away from the lowest order value, respectively. As for the potential B, it gives almost converged results within the $K_{\max }=14$ space only for $N \leqslant 9$. Starting with $N=10$ the convergence deteriorates, getting gradually worse with increasing $N$. The worst deviation from the $K_{\max }=0$ value, about $34 \%$, occurs for $N=112$. Then the convergence slightly improves.

The different rate of convergence can be understood by examining the evolution of nondiagonal couplings with $N$. The nondiagonal potentials $U_{00,41}(\rho)$ and $U_{00,42}(\rho)$ that couple $\chi_{K=0}(\rho)$ with two $\chi_{K=4, \gamma}(\rho)$ channel functions are shown in Fig. 1 for $N=10,112$, and 728 for both potentials $\mathrm{A}$ and B. For the set A and for $N=10$ the nondiagonal couplings $U_{00,4 \gamma}(\rho)$ are indeed small as compared to the diagonal potential $U_{00}(\rho)$ in the main, $K=0$, channel, as expected. The $U_{00}(\rho)$ is negative for all $\rho$, however, due to the repulsive three-body force; the attraction at small $\rho$ gets weaker [see Fig. 1(a)]. This does not matter much because this region of $\rho$ is dominated by the centrifugal barrier and the channel wave functions $\chi_{K \gamma}(\rho)$ are very small. However, for potential $\mathrm{B}$, where the repulsive core has a shorter range and a larger strength, the total potential $U_{00}(\rho)$ becomes repulsive almost everywhere except for a small pocket around $\rho \sim 25$ a.u. [Fig. 1(b)]. Although the nondiagonal couplings are small in comparison to $U_{00}(\rho)$ at those $\rho$ where this $U_{00}(\rho)$ is large, they have nodes at different locations than $U_{00}(\rho)$ does so that the their tails are nonnegligeable in the attractive pocket, which makes the convergence for potential B much worse than for A. With increasing $N$ the contribution from the repulsive three-body potential increases as $N ! /(N-3) ! / 3$ ! at small $\rho$, which is much faster than the $N ! /(N-2) ! / 2$ ! increase of the contribution from the attractive two-body potential. At the same time the three-body potentials decrease with $\rho$ as $\rho^{-6}$, which is faster than the $\rho^{-3}$ decrease of the contributions from the two-body attractive potentials. This results in a similar picture to the case of $N=10$ with the potential $\mathrm{B}$, where the depth of the attractive potential well is negligible with respect to the magnitude of the repulsive core [see Fig. 1(c)-(f)]. The repulsive core in nondiagonal coupling potentials is much smaller than in $U_{00}(\rho)$, and it does become smaller with $N$. However, the tails of nondiagonal potentials leak into the area of the attractive potential well, thus affecting the convergence. A more detailed study has revealed that it is the absolute values of the nondiagonal couplings around the minimum of $U_{00}(\rho)$ rather than their values near the nodes of $U_{00}(\rho)$ that are mainly responsible for the convergence. It was found that it is sufficient to solve the coupled system of differential equations (3) only in a very restricted region of $\rho$ in order to get very precise (within seven digits) ground and first excited state binding energies. The absolute values of the couplings in this restricted region determine the convergence. For example, for $N=112$ with set B it is sufficient to narrow the interval of $\rho$ by $160 \leqslant \rho \leqslant 200$ a.u. to get a six-digit accuracy for the binding energy. The strength of the couplings here is about $3 \%$ at the bottom of the attractive pocket, while at $\rho \sim 160$ a.u. they are about $4 \%$ and $13 \%$ of $U_{00}(\rho)$. The change in the binding energy is $3 \%$ when increasing $K_{\max }$ from 0 to 4 . A further increase of $K_{\text {max }}$ produces many couplings of a 3-5\% strength of $U_{00}(\rho)$, and this leads to further small (about 1\%) changes of the 

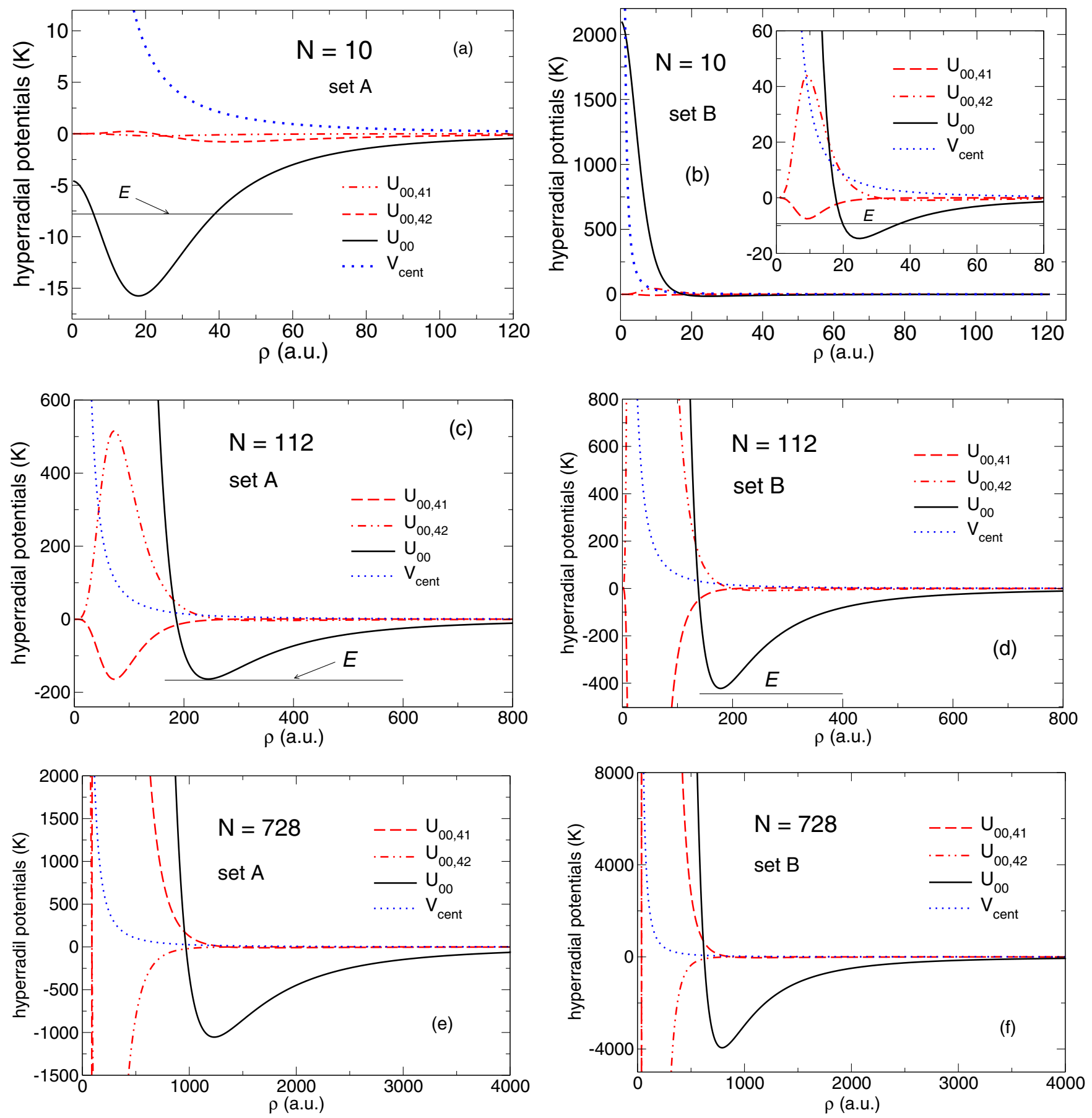

FIG. 1. (Color online) Two nondiagonal hyperadial potentials $U_{00, K=4 \gamma}(\rho)$ in comparison to the lowest order potential $U_{00}(\rho)$, centrifugal potential $V_{\text {cent }}=\frac{\hbar^{2}}{2 m} \mathcal{L}_{0}\left(\mathcal{L}_{0}+1\right) / \rho^{2}$ and the ground state energy $E$ for $N=10(a, b), N=112(c, d)$, and $N=278(e, f)$ atoms of helium calculated with potential $\mathrm{A}(a, c, e)$ and $\mathrm{B}(b, d, f)$.

binding energy with $K_{\max }$. The situation is better for $N=728$ with the same potential. Here it is sufficient to solve Eq. (3) in the interval $730 \leqslant \rho \leqslant 830$ a.u. to get an accuracy of six digits. In this interval the nondiagonal couplings do not exceed $1.5 \%$ of $U_{00, K \gamma}$ and the convergence seems to be slightly faster than for $N=112$. It is possible that it will improve further with increasing $N$. No attempts were undertaken to check this because for larger $N$ a new algorithm to calculate confluent hypergeometrical functions that make $U_{K \gamma, K^{\prime} \gamma^{\prime}}(\rho)$ should be developed.
For almost all first excited $0^{+}$states the $K_{\max }=14$ model space is not sufficient to get a converged value of the binding energy. However, the calculated separation energies of single helium atoms, $E_{0}(N-1)-E_{1}(N)$, are more stable with respect to the increase of $K_{\max }$ so that in most cases it is possible to make a conclusion where these states are bound or not. Thus, for potential A the first excited $0^{+}$states are bound for all $N$ and are attached to the binding energies of the ground states of $N-1$ atoms, while the second excited $0^{+}$ states are unbound, at least for $N \leqslant 240$, following a universal 
TABLE II. Absolute values of binding energies (in K) of excited states of 10, 20, and 728 helium atoms obtained with potential A and shown for several values of $K_{\max }-K_{\min }$. In these particular cases $K_{\min }$ coincides with the angular momentum of the state. In the last column the ground state binding energy of 727 atoms is shown to indicate where the first threshold in $N=728$ is located.

\begin{tabular}{|c|c|c|c|c|c|c|c|c|c|c|c|}
\hline \multirow[b]{2}{*}{$K_{\max }-K_{\min }$} & \multirow{2}{*}{$\frac{N=10}{2_{1}^{+}}$} & \multirow{2}{*}{$\frac{N=20}{2_{1}^{+}}$} & \multicolumn{8}{|c|}{$N=728$} & \multirow{2}{*}{$\frac{N=727}{0_{1}^{+}}$} \\
\hline & & & $2_{1}^{+}$ & $2_{2}^{+}$ & $2_{3}^{+}$ & $4_{1}^{+}$ & $4_{2}^{+}$ & $4_{3}^{+}$ & $3_{1}^{-}$ & $3_{2}^{-}$ & \\
\hline 0 & 6.1940 & 21.440 & 1036.9 & 1036.2 & 1035.5 & 1036.9 & 1036.2 & 1036.0 & 1036.4 & 1035.7 & 1035.5 \\
\hline 2 & 6.2221 & 21.508 & 1037.5 & 1036.9 & 1036.8 & 1037.8 & 1037.1 & 1036.8 & 1037.2 & 1036.5 & 1035.5 \\
\hline 4 & 6.3099 & 21.754 & 1953.0 & 1052.3 & 1051.6 & 1053.0 & 1052.3 & 1052.1 & 1052.5 & 1051.8 & 1051.6 \\
\hline 6 & 6.3251 & 21.855 & 1062.5 & 1061.8 & 1061.1 & 1062.5 & 1061.9 & 1061.6 & 1062.0 & 1061.3 & 1061.0 \\
\hline 8 & 6.3340 & 21.902 & 1071.4 & 1070.7 & 1069.9 & 1071.4 & 1070.7 & 1070.4 & 1070.9 & 1070.2 & 1069.9 \\
\hline 10 & 6.3369 & 21.920 & 1079.9 & 1079.2 & 1078.5 & $1079 . \underline{8}^{\mathrm{a}}$ & $1078 . \underline{8}^{\mathrm{a}}$ & $1078 . \underline{3}^{\mathrm{a}}$ & $1079 . \underline{3}^{\mathrm{a}}$ & $1077 . \underline{8}^{\mathrm{a}}$ & 1078.5 \\
\hline 12 & 6.3384 & 21.928 & $1087 . \underline{6}^{\mathrm{a}}$ & & & & & & & & 1086.3 \\
\hline
\end{tabular}

a Only a harmonic oscillator expansion method of solving Eq. (3) was used to calculate the binding energies for $K_{\max }=14$, but this method becomes less accurate for $N=728$. The underlined fifth digit most likely should be two units larger for $2_{1}^{+}, 4_{1}^{+}$, and $3^{-}$states, based on comparison with finite difference method calculations for smaller $K_{\max }$, while for $4_{2}^{+}$and $4_{3}^{+}$it may be four and seven units larger, respectively.

picture observed for $N \leqslant 16$ body systems in calculations with two-body interactions only [10-13]. For $N=728$ the second excited $0^{+}$state seem to become bound, but a precision of at least seven digits is required to confirm this. For potential B, the first excited $0^{+}$states for $4 \leqslant N \leqslant 7$ are unbound within the model space used. However, exponential extrapolation suggests that some of them may be bound. For $N \geqslant 8$ the first excited $0^{+}$states become bound and at $N \geqslant 240$ the second excited $0^{+}$state become bound as well.

\section{STATES WITH NONZERO ANGULAR MOMENTUM}

Although for realistic states with nonzero angular momentum the $l$ dependence of the atom-atom interaction could be important, the convergence study performed with $l$-independent interatomic forces of type A or B would be still useful. Most of these states will be unbound. Since the convergence of binding energy could be achieved only for bound states, in this section their search has been undertaken using potential set A. It was found that the $N \leqslant 9$ systems do not have any bound excited states with nonzero angular momentum. The first $2^{+}$state becomes bound at $N \geqslant 10$, and its energy has been practically converged by $K_{\max }=14$. The $2^{+}$state is also bound for $N=20$ where its energy still remains practically converged (see Table II). No other bound states with nonzero angular momentum are found in this area.

At large $N$ one can expect more bound states to exist. However, for $N>20$ the convergence of the binding energies deteriorates, and at large $N$ it becomes difficult to draw definite conclusions about how many bound states are there. To illustrate this point, Table II shows the three lowest $2^{+}$, $4^{+}$, and two lowest $3^{-}$states as a function of $K_{\max }-K_{\min }$, where $K_{\min }$ is determined by the angular momentum. The first $N=727$ threshold is shown there as well. One can see that if the energies of these states were plotted as functions of $K_{\max }-K_{\min }$, then the three $2^{+}$and $4^{+}$states, as well as the two $3^{-}$states, would appear bound. However, if they were plotted as functions of $K_{\max }$, only then would all these states appear unbound. This happens because the change of energy with increasing $K_{\max }$ is comparable with the excitation energies themselves. The exponential extrapolation does not help to find out if these states are bound because the errors associated with this extrapotation are larger than the excitation energies themselves. Achieving convergence of binding energies is crucial for making a final conclusion about the number of bound excited states with nonzero angular momentum.

\section{SUMMARY AND CONCLUSIONS}

The numerical calculations with a soft attractive two-body $\mathrm{He}-\mathrm{He}$ potential and a repulsive three-body He-He-He hypercentral potential have shown that the improved convergence of the hyperspherical-harmonics expansion with $N$, originally noticed in Ref. [2] for $N=4,5,6$ and then explained in Ref. [1], occurs only for a relatively small number of $N$ when three-body forces are included. The interval of $N$ where this convergence is fast is determined by the range of the three-body repulsion. For a long-range three-body force the convergence starts deteriorating around $N=20$, while for a short-range three-body force this deterioration begins earlier, at $N=10$. Convergence remains reasonably fast while the total hyperradial diagonal potential remains attractive at small $\rho$. It gets worse when the $N ! /(N-3) ! / 3$ ! increase from the contribution of the three-body repulsion starts to dominate the two-body attraction at small $\rho$ and the tails of nondiagonal potentials leak into the shallow attractive pocket shaped by the interference between two-body attraction and three-body repulsion. The convergence becomes slow around $N=112$ but seems to improve near $N=728$. Table III shows the difference between the binding energies obtained in the $K_{\max }=0$ and $K_{\max }=14$ approximations for $4 \leqslant N \leqslant 728$, where they are also compared to the calculations of other methods.

The long range of potential A has been suggested in Ref. [2] assuming its link to the range of two-body force via relation $\rho_{0}=\sqrt{2} r_{0}$. However, as seen from Table III, this potential underbinds helium clusters for $N>6$ and this underbinding becomes severe for $N>10$. To improve the description of binding energies, a shorter range three-body force is required, inconsistent with $\rho_{0}=\sqrt{2} r_{0}$. This may mean that in realistic systems either the physical (other than induced by neglecting repulsive two-body cores) three-body force has a short range or contributions from other orders become important. In particular, one can imagine that an attractive 
TABLE III. Absolute values of binding energies (in $\mathrm{K}$ ) of $N$ helium atoms obtained in the $K=0\left(\mathrm{HH}_{0}\right)$ and $K=14\left(\mathrm{HH}_{14}\right)$ approximations with two sets of potentials, (A) and (B), that include a phenomenological soft attractive two-body potential plus a three-body repulsive potential. These energies are compared to diffusion Monte Carlo (DMC) calculations [7], Monte Carlo hyperspherical (MCH) calculations [8], and the Green's function Monte Carlo (GFMC) and the variational Monte Carlo (VMC) calculations [9] with hard-core He-He potential.

\begin{tabular}{|c|c|c|c|c|c|c|c|c|}
\hline$N$ & $\mathrm{HH}_{0}(\mathrm{~A})$ & $\mathrm{HH}_{14}(\mathrm{~A})$ & $\mathrm{HH}_{0}(\mathrm{~B})$ & $\mathrm{HH}_{14}(\mathrm{~B})$ & DMC & $\mathrm{MCH}$ & GFMC & VMC \\
\hline 4 & 0.5389 & 0.5688 & 0.5532 & 0.5869 & 0.5584 & 0.5569 & 0.5334 & 0.512 \\
\hline 5 & 1.2881 & 1.3266 & 1.3122 & 1.3778 & 1.3022 & 1.2965 & & \\
\hline 6 & 2.2938 & 2.3389 & 2.3372 & 2.4689 & 2.3194 & 2.3095 & & \\
\hline 7 & 3.4863 & 3.5361 & 3.5831 & 3.8291 & 3.5641 & 3.5657 & & \\
\hline 8 & 4.8040 & 4.8569 & 5.0164 & 5.4324 & 5.0374 & 5.0203 & 4.9325 & 4.776 \\
\hline 9 & 6.1995 & 6.2543 & 6.6117 & 7.2572 & 6.6765 & 6.6779 & & \\
\hline 10 & 7.6393 & 7.6959 & 8.3501 & 9.2851 & 8.5010 & 8.4952 & & \\
\hline 20 & 22.286 & 22.738 & 31.316 & 37.161 & & & 32.546 & 31.4 \\
\hline 40 & 50.902 & 54.893 & 94.850 & 111.62 & & & 99.492 & 95.84 \\
\hline 70 & 93.530 & 103.07 & 212.83 & 244.86 & & & 218.68 & 211.4 \\
\hline 112 & 153.29 & 168.86 & 402.28 & 454.07 & & & 403.2 & 394.24 \\
\hline 240 & 336.13 & 364.71 & 1059.9 & 1162.5 & & & & 1105.6 \\
\hline 728 & 1037.0 & 1095.1 & 3903.0 & 4137.5 & & & & 3603.6 \\
\hline
\end{tabular}

contribution from an induced four-body force, presented, for example, by a simple hypercentral Gaussian, could become important with $N$ as it should grow as $N ! /(N-4) ! / 4$ ! at small $\rho$ while decreasing as $\rho^{-9}$ at large $\rho$. It could remove the strong repulsive core associated with the three-body repulsion and, therefore, decrease the coupling potentials thus improving the convergence with $N$. The danger is that at some $N$ this term could create a strong unphysical attractive pocket in the area which should be dominated by the centrifugal potential thus generating unphysical states. In this case the next order (five-body force) repulsive contributions could be needed to remove this effect. While the role of many-body forces is certainly worth investigating, the need for a shorter range of the three-body force can be simply explained by the van der Waals two-body correlations. It was shown in Ref. [6] that in the Efimov regime the van der Waals length universally determines three-body repulsion with the range of $2 R_{\mathrm{vdW}}$. For helium clusters this value is very close to $\rho_{0}$ from set $\mathrm{B}$. Thus if the the range of the three-body repulsion in set $B$ is microscopically justified, then many-body calculations without explicit consideration of two-body correlations, of the type presented here, will always need larger model spaces to obtain converged solutions.

Finally, convergent solutions are important for making definite conclusions about the number of bound excited states. With the long-range three-body force the converged solutions for the first $2^{+}$state have been found only for $10 \leqslant N \leqslant 20$. For $N>20$, where the convergence deteriorates, the interpretation of their binding becomes ambiguous. Convergence accelerating methods are needed to make reliable predictions for spectra of systems with large numbers of atoms.

\section{ACKNOWLEDGMENT}

Support from the the UK STFC ST/J000051/1 grant is acknowledged.
[1] N. K. Timofeyuk, Phys. Rev. A 86, 032507 (2012).

[2] M. Gattobigio, A. Kievsky, and M. Viviani, Phys. Rev. A 84, 052503 (2011).

[3] Yu. F. Smirnov and K. V. Shitikova, Sov. J. Part. Nucl. 8, 344 (1977).

[4] N. K. Timofeyuk, Phys. Rev. C 65, 064306 (2002).

[5] N. K. Timofeyuk, Phys. Rev. C 78, 054314 (2008).

[6] P. Naidon, S. Endo, and M. Ueda, Phys. Rev. A 90, 022106 (2014).

[7] M. Lewerenz, J. Chem. Phys. 106, 4596 (1997).

[8] D. Blume and C. H. Greene, J. Chem. Phys. 112, 8053 (2000).
[9] V. R. Pandharipande, J. G. Zabolitzky, S. C. Pieper, R. B. Wiringa, and U. Helmbrecht, Phys. Rev. Lett. 50, 1676 (1983).

[10] A. Deltuva, R. Lazauskas, and L. Platter, Few-Body Syst. 51, 235 (2011).

[11] M. Gattobigio, A. Kievsky, and M. Viviani, Phys. Rev. A 86, 042513 (2012).

[12] A. Kievsky, M. Gattobigio, and N. K. Timofeyuk, Few-Body Syst. 55, 945 (2014).

[13] A. Kievsky, N. K. Timofeyuk, and M. Gattobigio, Phys. Rev. A 90, 032504 (2014). 